\title{
ANÁLISE DO REATOR DO TIPO TANQUE AGITADO (CSTR): COMPORTAMENTO DINÂMICO NA PARTIDA E ESTABILIDADE DA OPERAÇÃO ESTACIONÁRIA
}

\author{
M. V. de C. MOTA ${ }^{1}$, F. T. VIEIRA ${ }^{1}$ \\ ${ }^{1}$ LAMCES - Laboratório de Métodos Computacionais, Controle e Estimação \\ Universidade Federal do Espírito Santo - Engenharia Química (campus Alegre) \\ E-mail para contato: marcosvcm13@gmail.com
}

\begin{abstract}
RESUMO - No meio industrial, o reator tipo tanque agitado (CSTR) é amplamente utilizado, em processos que normalmente ocorrem em estado estacionário e de forma não isotérmica, sendo processadas grandes quantidades de carga. Entretanto, pouca atenção é dada à etapa de partida do reator. As equações de projeto do reator CSTR recaem em sistemas complexos de equações algébricas e/ou diferenciais, o que torna uma resolução trabalhosa por meios analíticos (quando estas existem). Assim, o uso de recursos computacionais é uma poderosa ferramenta para se avaliar o comportamento dinâmico do sistema durante a partida do reator e as melhores condições de operação em estado estacionário. O processo avaliado é a hidrólise do óxido de propileno em propilenoglicol, o qual é utilizado como umectante na indústria alimentícia, fluido de refrigeração, solvente, dentre outros. Uma questão importante é que o reator pode atingir momentaneamente temperaturas muito elevadas antes de atingir o estado estacionário, o que resulta em perdas consideráveis de reagente por evaporação, daí a necessidade de ser avaliar a dinâmica da partida do reator. Além disso, a operação estacionária pode apresentar multiplicidade de estados estacionários. Uma forma de avaliar a estabilidade de forma simples é a partir da análise dos autovalores da matriz jacobiana, construída a partir dos balanços de massa e energia. Tal metodologia permite avaliar de forma direta em que condições do processo a operação é estável.
\end{abstract}

\section{INTRODUÇÃO}

Fatores como a alta competitividade, maior diversidade de produtos e menor disponibilidade de matéria prima, forçam as indústrias a otimizarem seu processos. Porém, ajustes feitos no meio industrial exigem muito tempo e recursos, além de promover grande risco aos envolvidos na mudança. Neste cenário, a modelagem e simulação de processos é uma alternativa mais simples e segura para aperfeiçoar um processo.

A representação dos processos através de equações matemáticas é conhecida como modelagem do processo. Quanto mais próximo da realidade, mais complexos serão os modelos matemáticos propostos para tal tarefa (SCHUTZ et al, 2013).

Um dos equipamentos de maior influência no rendimento de processos é o reator, mais especificamente para este trabalho, o reator tipo tanque perfeitamente misturado (CSTR). Este tipo de reator é usado principalmente em reações em fase líquida, onde é normalmente operado em estado estacionário e é feita a consideração de perfeitamente misturado, 
consequentemente, a temperatura, concentração e a velocidade de reação não dependem do tempo nem da posição dentro do reator (FOGLER, 2009).

Há muitos fatores que podem exercer influência em um processo com reação química, como por exemplo, a temperatura, pressão, energia de ativação, concentração dos reagentes, a energia cedida ou retirada pela reação $\left(\Delta \mathrm{H}_{\text {reação }}\right)$, entre outros. Neste trabalho, serão avaliados como as condições iniciais de temperatura e concentração do reagente interferem na dinâmica da reação, verificando também se existe algum intervalo do processo onde a reação se torne inviável (como por exemplo, aumento excessivo da temperatura causando vaporização de reagente).

Nesse tipo de processo é possível verificar a existência de múltiplos estados estacionários, que podem ser estáveis ou não (PEREIRA e OLIVEIRA, 2006). Nesse sentido, para a analise de estabilidade será utilizado o método de matriz jacobiana apresentado em RAWLINGS e EKERDT (2002).

\section{CARACTERÍSTICAS DO PROCESSO E MODELAGEM}

Para apresentar a metodologia de análise da dinâmica da partida do reator e seu comportamento em estado estacionário, foi escolhido o processo de hidrólise do óxido de propileno em propilenoglicol (Figura 1) em um reator CSTR com jaqueta, devido a grande importância e versatilidade do produto no mercado e a facilidade de encontrar parâmetros intrínsecos da reação na literatura.

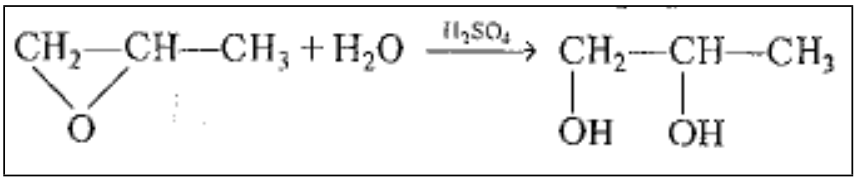

Figura 1 - hidrólise do Óxido de Propileno

O trocador de calor tipo jaqueta se torna necessário, pois o óxido de propileno se volatiliza a temperaturas acima de $180^{\circ} \mathrm{F}$, fazendo desta a temperatura limite de operação do processo, uma vez que acima desta temperatura a volatização resulta em elevada perda de reagente. Para simular o processo, foram implementados no software MATLAB os balanços de massa (EQ1) e energia (EQ2 e EQ3), utilizando os parâmetros e condições iniciais do processo listados na Tabela 1.

$$
\begin{aligned}
& \frac{C_{A 0}-C_{A}}{\tau}+r_{A}=\frac{d C_{A}}{d t} \\
& \frac{d T}{d t}=\frac{Q-F_{A 0} \sum \theta_{i} C p_{i}\left(T-T_{0}\right)+\left(\Delta H_{R}\right)\left(\mathrm{r}_{\mathrm{A}} \mathrm{V}\right)}{\sum N_{i} C p_{i}} \\
& Q=m_{w} C p_{w}\left(\mathrm{~T}_{\mathrm{a} 1}-\mathrm{T}\right)\left[1-\exp \left(-\frac{U A}{m_{w} C p_{w}}\right)\right]
\end{aligned}
$$

Tabela 1 - Parâmetros e condições inicias (FOGLER, 2009) 


\begin{tabular}{|c|c|c|c|}
\hline $\begin{array}{l}\text { Congresso Brasileiro de Engenharła } \\
\text { Quimica em Iniciação Científica }\end{array}$ & \multicolumn{3}{|c|}{$\begin{array}{r}\text { XI Congresso Brasileiro de Engenharia } \\
\text { Química em Iniciação Científica } \\
\text { Unicamp - Campinas - SP } \\
19 \text { a } 22 \text { de julho de } 2015\end{array}$} \\
\hline Condição inicial & Símbolo & Valor & Unidade \\
\hline $\begin{array}{l}\text { Calor específico da mistura } \\
\text { (calculado) }\end{array}$ & Com & 284,37 & $\mathrm{btu} / \mathrm{lbmol}$ de $\mathrm{A} .{ }^{\circ} \mathrm{F}$ \\
\hline Calor específico do refrigerante & $\mathrm{C}_{\mathrm{pi}}$ & 18 & $\mathrm{btu} / \mathrm{lbmol} .^{\circ} \mathrm{F}$ \\
\hline Calor gerado pela reação & $\Delta \mathrm{H}_{\mathrm{r}}$ & 36000 & btu/lbmol de A \\
\hline Coeficiente global de troca térmica & UA & 16000 & btu \\
\hline Temperatura inicial & $\mathrm{T}_{0}$ & 75 & ${ }^{\circ} \mathrm{F}$ \\
\hline Temperatura inicial do Refrigerante & $\mathrm{T}_{\mathrm{a} 1}$ & 60 & ${ }^{\mathrm{o}} \mathrm{F}$ \\
\hline Vazão de Óxido de Propileno & $\mathrm{F}_{\mathrm{A}}$ & 80 & $\mathrm{lbmol} / \mathrm{h}$ \\
\hline Vazão de Água & $\mathrm{F}_{\mathrm{B}}$ & 1000 & $\mathrm{lbmol} / \mathrm{h}$ \\
\hline Vazão de inerte & $\mathrm{F}_{\mathrm{C}}$ & 100 & $\mathrm{lbmol} / \mathrm{h}$ \\
\hline Vazão de Refrigerante & $\mathrm{F}_{\text {ref }}$ & 1000 & $\mathrm{lbmol} / \mathrm{h}$ \\
\hline Volume reator & V & 500 & gal \\
\hline
\end{tabular}

Para análise de estabilidade do estado estacionário, determinou-se a matriz jacobiana do processo, a partir da linearização das equações diferenciais de balanço de massa $\left(f_{1}\right)$ e energia $\left(\mathrm{f}_{2}\right)$ por expansão de Série de Taylor, em torno da temperatura estacionária $\left(\mathrm{T}_{\mathrm{s}}\right)$ e concentração estacionária $\left(\mathrm{Ca}_{\mathrm{s}}\right)$ (RAWLINGS e EKERDT, 2002). A matriz jacobiana é representada pela Equação 3.

$$
J=\left(\begin{array}{ll}
\frac{\partial f_{1}}{\partial C_{A}} & \frac{\partial f_{1}}{\partial T} \\
\frac{\partial f_{2}}{\partial C_{A}} & \frac{\partial f_{2}}{\partial T}
\end{array}\right)
$$

Para o sistema avaliado, a matriz jacobiana assume a forma apresentada na Equação 4:

$$
J=\left(\begin{array}{cc}
-\frac{1}{\tau}-K & K C_{A s} \frac{E}{T_{s}^{2}} \\
-\frac{\Delta H_{r}}{C_{p m}} K V & -\frac{F_{A 0} \sum_{i=1}^{n} \theta_{i} C_{P i}}{C_{p m}}-\frac{\Delta H_{r}}{C_{p m}} K C_{A s} V \frac{E}{T_{s}^{2}}
\end{array}\right)
$$

A matriz jacobiana permite uma análise simples de como as variáveis do processo influenciam na operação do sistema, uma vez que apenas o cálculo dos autovalores da matriz permite definir se a operação em estado estacionário é estável ou não. Quando os autovalores são ambos negativos, o sistema opera de forma estável. Quando a parte real é nula ocorre a chamada oscilação sustentada, e quando tem-se parte real positiva, a oscilação tende a aumentar deixando o sistema instável. 


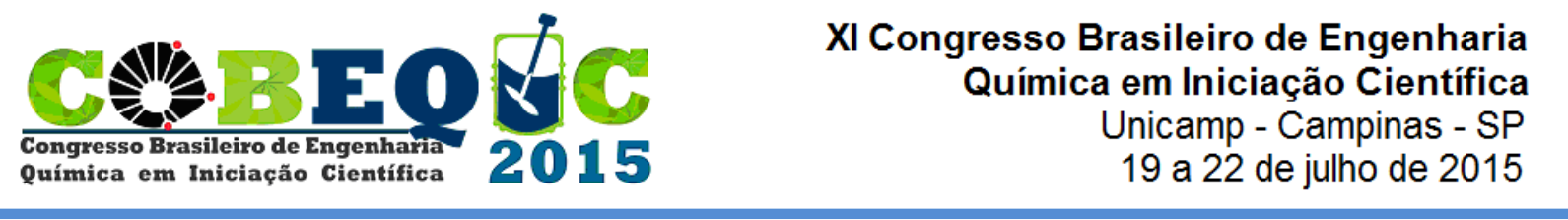

\section{RESULTADOS E DISCUSSÃO}

Na Figura 2 estão representados os resultados da simulação do processo baseados nos dados descritos na Tabela 1. Pode-se notar, através da Figura 2.a e 2.b, que o sistema atinge o estado estacionário após aproximadamente 2 horas. Além disso, nota-se também que durante a primeira hora do processo ocorrem mudanças consideráveis na concentração do reagente e na temperatura do processo. Isso se deve ao fato de que inicialmente o reator está preenchido apenas com água e à entalpia do processo, resultando na sobreelevação da temperatura em relação ao estado estacionário, fato que já foi apontado como desfavorável ao processo. Com o aumento da temperatura, aumenta-se também a velocidade da reação, fazendo com que o consumo de reagente seja elevado, diminuindo assim a concentração de óxido no reator. Após este primeiro momento, o processo vai se reajustando até alcançar seu equilíbrio.
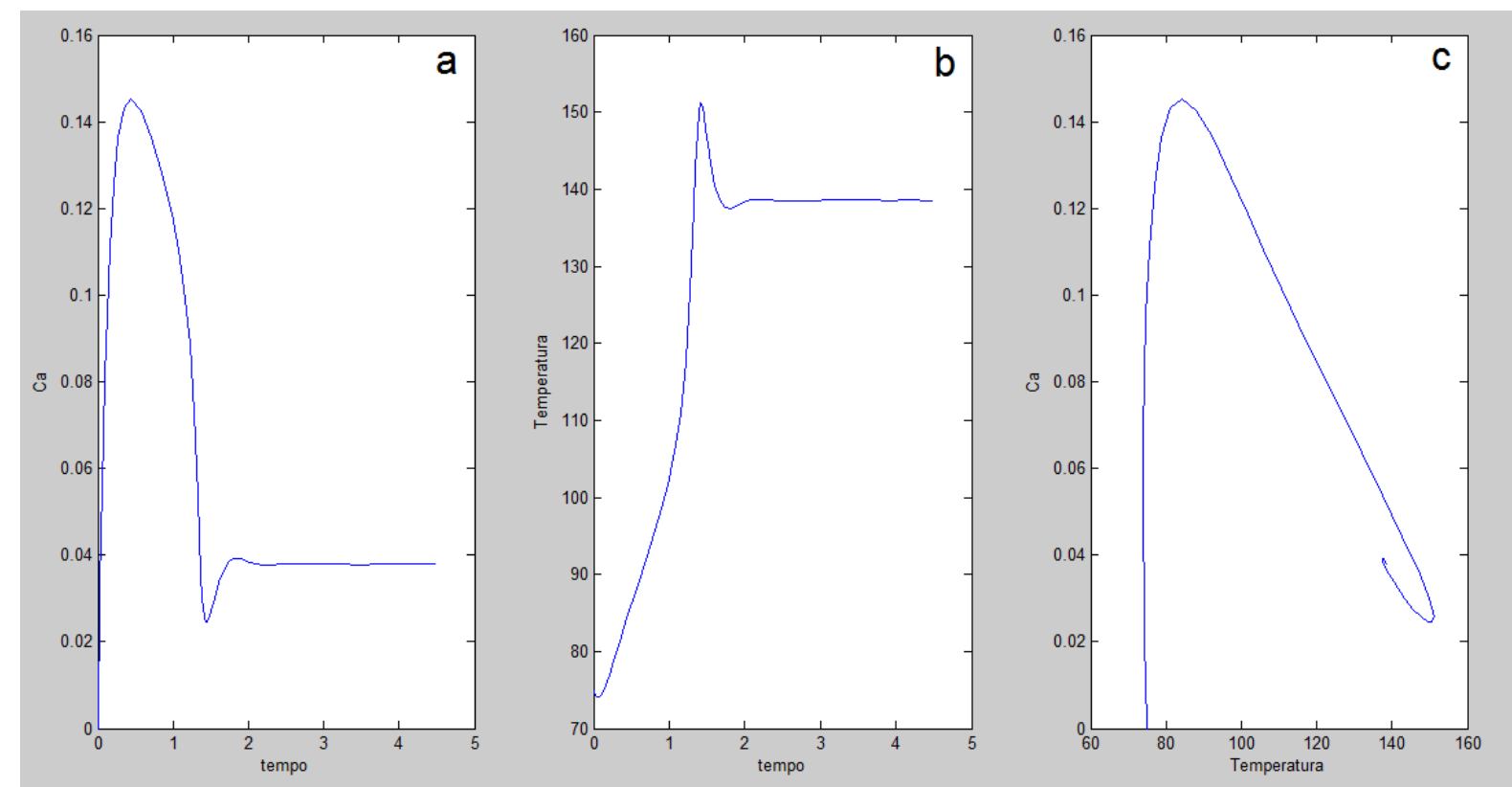

Figura 2 - a) Gráfico Concentração de A x Tempo/ b) Temperatura x Tempo/ c) Concentração de A x Temperatura.

A figura 2.c apresenta a trajetória do plano de fase concentração $\mathrm{x}$ temperatura, o qual mostra claramente a dinâmica do processo.

De forma a avaliar como as condições de partida do reator influenciam na dinâmica do processo foi realizado um mapeamento. Para tal, foi criado um algoritmo que gera, aleatoriamente, pares de concentração de reagente e temperatura inicial com intuito de observar a dinâmica inicial do processo e se este sistema recaí em outro estado estacionário. A Figura 3 mostra que independente das condições iniciais de temperatura e concentração avaliadas, o sistema sempre alcançou o mesmo ponto estacionário (ponto P). Entretanto, observa-se claramente que a concentração inicial do reagente e a temperatura de alimentação deste podem resultar numa dinâmica do processo que resulta em um período de operação com temperaturas muito elevadas, o que resultará em elevada perda de reagente, uma vez que este apresenta altas taxas de evaporação acima de $180^{\circ} \mathrm{F}$. 

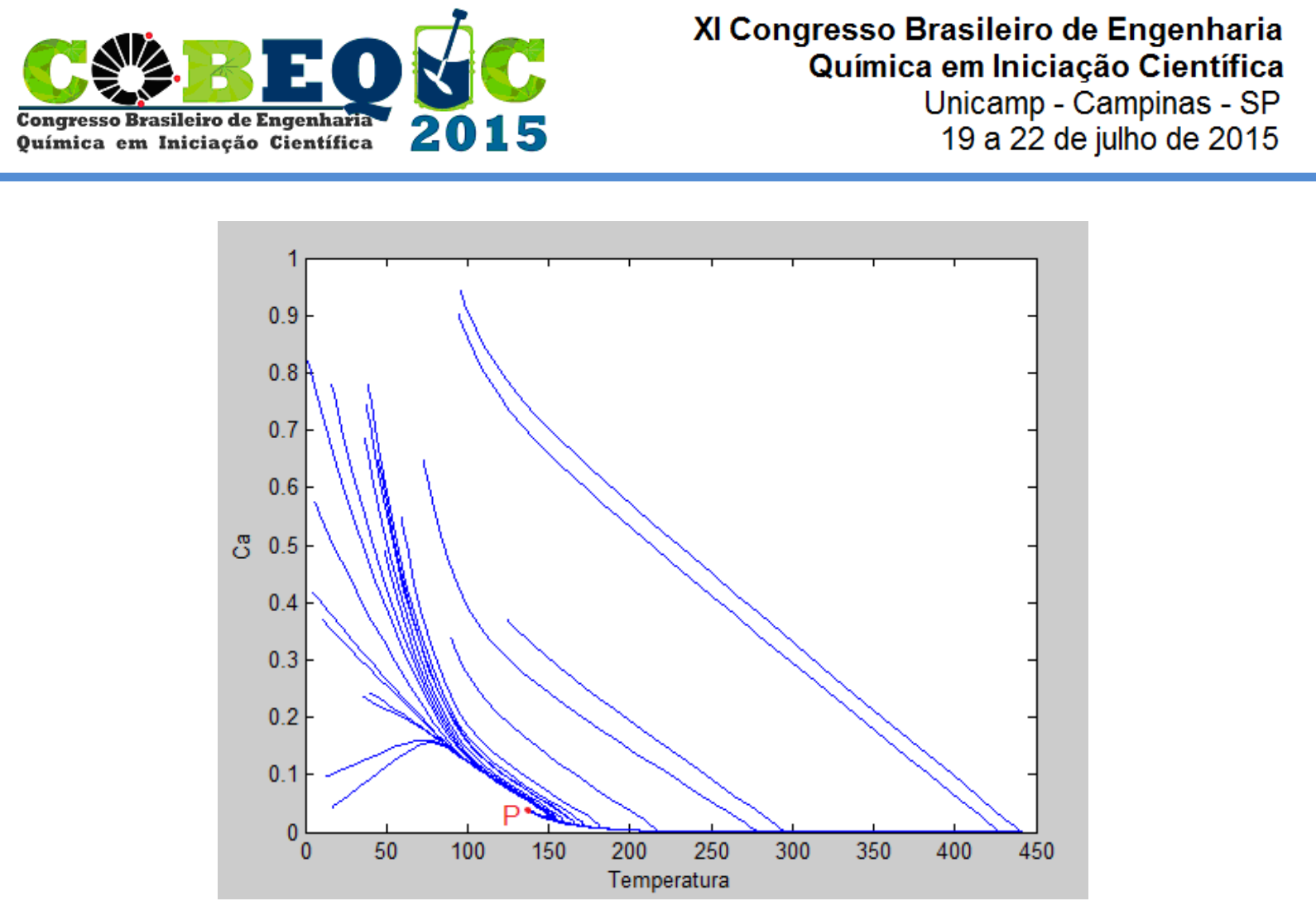

Figura 3 - Influência das condições iniciais de concentração de reagente e temperatura de alimentação sobre a partida do reator.

Apesar da dinâmica do processo apresentar trajetórias distintas, nas condições avaliadas o sistema é atraído para um único ponto estacionário, mas isto não garante a unicidade da condição estacionária, nem mesmo que o sistema não possa operar de forma instável caso a partida do reator seja realizada sob outras condições.

Para avaliar essa questão de forma simples, a metodologia adotada foi avaliar os autovalores da matriz jacobiana do sistema. No caso do mapeamento apresentado na Figura 3, os autovalores foram $-5,93$ e -2,01, confirmando assim a estabilidade da operação nessa condição (ponto $\mathrm{P}, 138^{\circ} \mathrm{F}$ ), uma vez que ambos os autovalores são reais e negativos.

Entretanto, sabe-se que reatores CSTR podem apresentar multiplicidade de estado estacionário. Baseado nos resultados apresentados na Figura 3, é possível que o sistema possa ser atraído por outras regiões de operação estacionária. Assim, realizou-se uma análise da influência da temperatura estacionária sobre os autovalores da matriz jacobiana, cujos resultados são apresentados na Figura 4. Pode-se perceber que abaixo de $152{ }^{\circ} \mathrm{C}$ e acima de $183{ }^{\circ} \mathrm{C}$ a operação é estável, havendo apenas uma pequena faixa de temperatura em que o reator pode operar de modo instável caso um sistema de controle não atue no sistema.

A metodologia de calcular a jacobiana é uma alternativa interessante de avaliar a estabilidade do sistema. Usualmente, nesse tipo de sistema se avalia a interseção das curvas de conversão em função da temperatura derivadas a partir do balanço de massa e de energia para se determinar a existência de múltiplos estados estacionários. Por outro lado, a utilização da matriz jacobiana, além de determinar que faixa de temperatura resulta na operação estável, permite avaliar de modo mais amplo como as demais variáveis do processo influenciam na estabilidade do processo, além de dar um indicativo da sensibilidade do sistema frente à mudanças nas condições de operação do processo, sendo esta sensibilidade proporcional ao autovalor. 

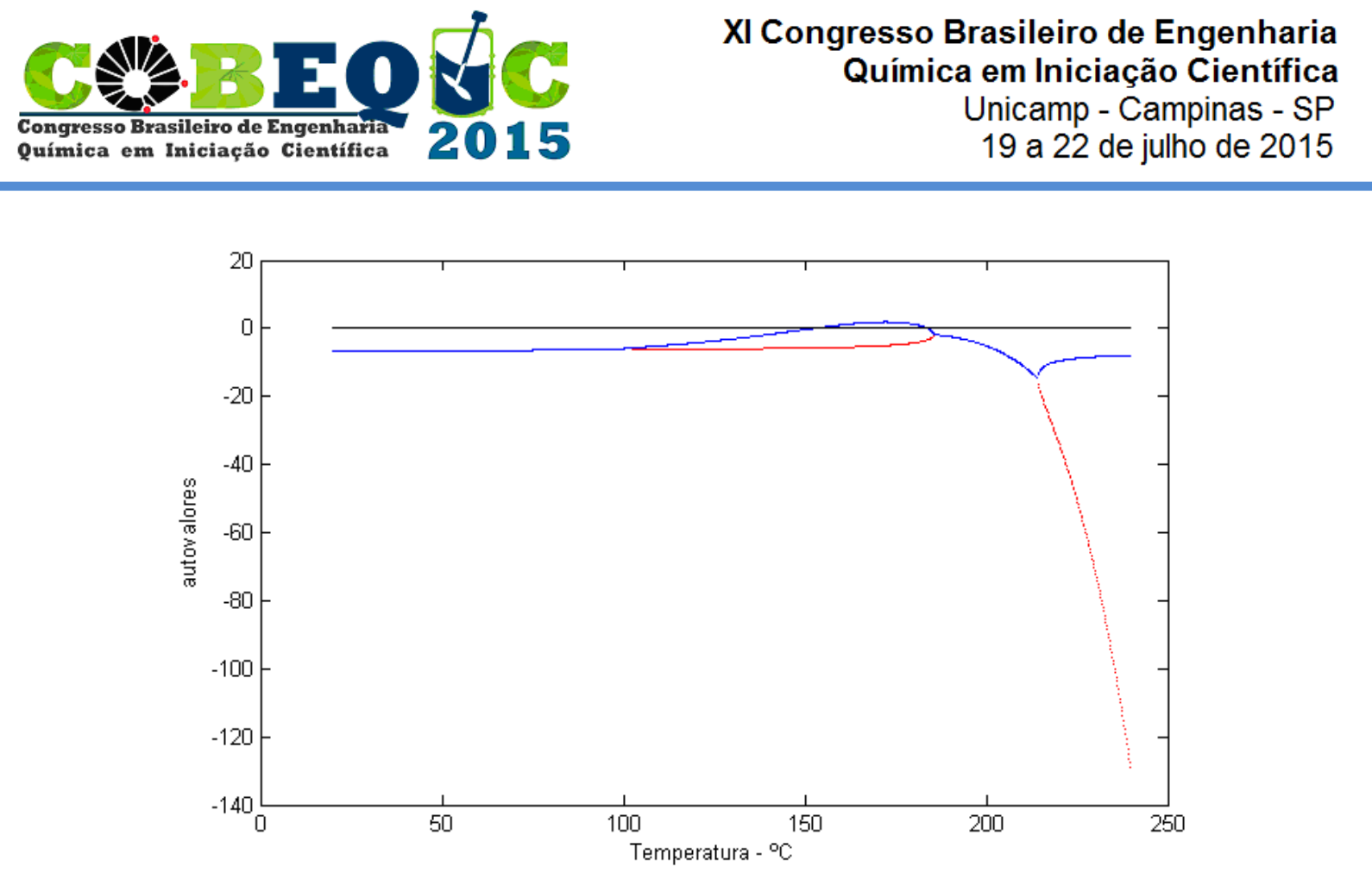

Figura 4 - Autovalores da matriz jacobiana em função da temperatura

\section{CONCLUSÃO}

Os resultados indicam que, em processos industriais, a análise da dinâmica durante a partida do reator CSTR é extremamente importante. Condições operacionais de partida que resultem em menor custo e/ou tempo para se atingir o estado estacionário podem resultar em uma trajetória dinâmica que resulta em perdas de reagente por volatilização, como é o caso, ou em degradação de material em reações em que os reagentes e/ou produtos são termossensíveis. Daí a importância de se mapear como as condições de partida do reator influenciam sua dinâmica. Por outro lado, avaliar a operação estacionária do reator CSTR não é menos importante. Mudanças nas condições do processo podem resultar em condições de operação estacionária diferentes das originais. A avaliação da matriz jacobiana permite avaliar como alterações nas variáveis de processo influenciam na estabilidade na operação do reator, além de possibilitar identificar que variáveis ou características da reação (energia de ativação ou entalpia, por exemplo) mais influenciam na dinâmica do processo.

\section{REFERÊNCIAS}

FOGLER, H. Scott. Elementos de Engenharia das Reações Químicas. 4a edição. Rio de Janeiro: Editora LTC, 2009.

PEREIRA, F. M.; OLIVEIRA, S. C. Análise da Existência e da estabilidade de Múltiplos Estados Estacionários para a Hidrólise Enzimática da sacarose em Reator CSTR. Apresentado ao XVI Congresso Brasileiro De Engenharia Química, Santos, 2006.

RAWLINGS, J. B.; EKERDT, J. G. Chemical Reactor Analysis and Design Fundamentals.1 st ed. Madinson, Wisconsin: Editor Nob Hill Publishing, 2002.

SCHUTZ, G.; LAUER, J.; LOPES, J. M.; RANGEL, R. S. Modelagem e Simulação de Reatores Químicos no EMSO e GNU OCTAVE. 2013. Monografia (Bacharel em Engenharia Química). Faculdades Integradas de Aracruz. Aracruz, Brasil, 2013. 